\title{
THE PRESCREENING OF RETINOPATHY OF PREMATURITY (ROP): A REAL FACT
}

\author{
A. Pérez-Muñuzuri, A. Baña-Souto, O. López-Suárez, M.L. Couce-Pico, J.M. Fraga-Bermúdez \\ Pediatría - Servicio de Neonatología, Hospital Clínico Universitario de Santiago, Santiago de Compostela, \\ Spain
}

Background and aims: The screening of ROP is based on identifying patients with any grade of retinopathy. We present a simple early method of screening to identify patients at high risk of developing ROP.

Methods: 5-year prospective study of 145 preterm infants less than $1500 \mathrm{~g}$ and/or 32 weeks gestational age or less. We evaluated several demographic and clinical variables and serum IGF1 levels in the $3^{\text {rd }}$ week of life. A multinomial logistic regression was made to determine significative variables associated to ROP.

Results: 39 patients (26.9\%) developed ROP (51.3\% grade 1, 30.8\% grade 2, 17.9\% grade 3$)$. Only sepsis (clinical, analytical and/or microbiological) in the first 3 weeks post-partum and the serum IGF1 levels in the third week of life, both independently of gestational age, are clearly related with ROP. Figure 1 represents the probability of ROP obtained from regression analysis.

Conclusions: Prescreening of ROP can be made at third week of life, which serves to identify patients at high risk of developing ROP and enables procedures to be focussed and carried out more thoroughly, with the aim of avoiding the disease or at least minimising the stage to which it develops.

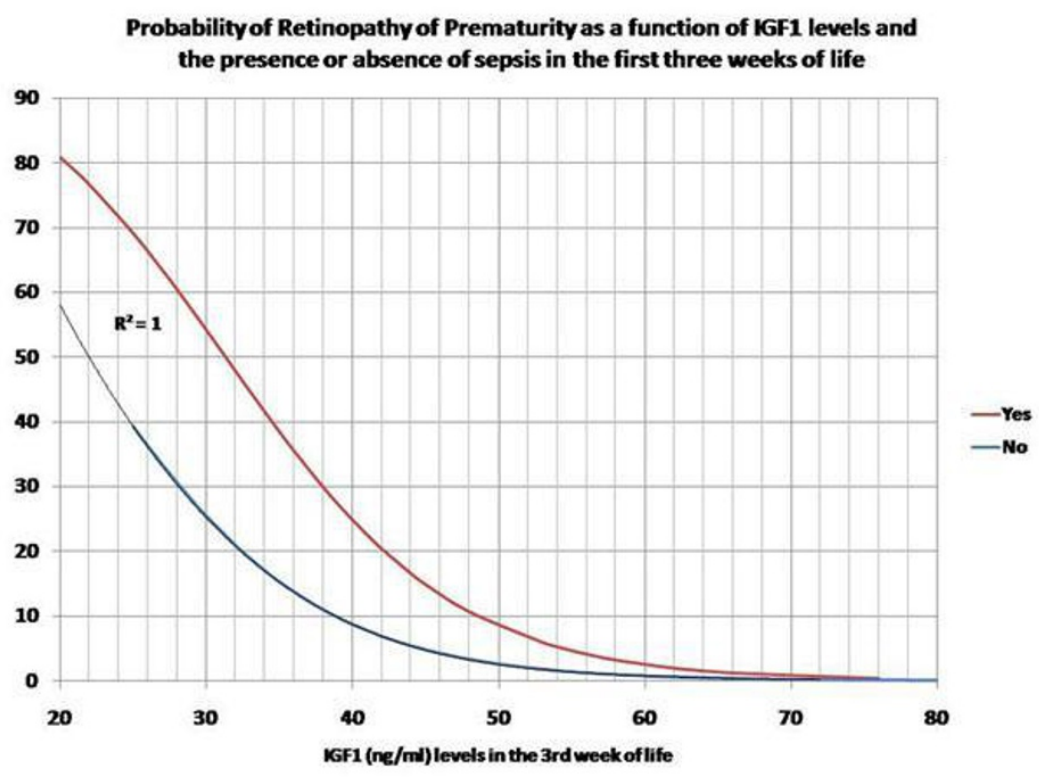

[Figure 1] 